\title{
Factors related to parental pre-treatment motivation in outpatient child and adolescent mental health care
}

\author{
Halewijn M. Drent ${ }^{1}$ (D) $\cdot$ Barbara van den Hoofdakker ${ }^{1} \cdot$ Annelies de Bildt $^{1} \cdot$ Jan K. Buitelaar ${ }^{2} \cdot$ Pieter J. Hoekstra $^{1}$. \\ Andrea Dietrich
}

Received: 1 August 2018 / Accepted: 16 August 2019 / Published online: 24 September 2019

(c) The Author(s) 2019

\begin{abstract}
The aim of this study was to investigate the relation between a variety of child, parent, family and environmental factors and pre-treatment motivation of parents of children and adolescents newly referred to a mental health care clinic in The Netherlands. Data were collected of 521 parents most involved in the upbringing of the child (443 mothers and 78 fathers; Dutch origin $97.1 \%$ ) of 207 girls and 314 boys (age $M=10.2$, range $1-18$ years). Treatment motivation was measured by the Parent Motivation Inventory. Least absolute shrinkage and selection operator (LASSO) regression was used to investigate the prediction strength of 33 factors in 5 domains: (1) source of referral and prior use of healthcare services, (2) child characteristics, (3) characteristics of the primary parent, (4) parenting characteristics of the primary parent, and (5) family characteristics. Twenty-one factors were statistically relevant, explaining $21.3 \%$ of the deviance in pre-treatment motivation. Child characteristics, mainly type and severity of problems, contributed most to the model. Notably, internalising problems contributed more than externalising problems. Furthermore, we found relations between parental pre-treatment motivation and parents' perceived self-efficacy, parents' perceived parenting competence, financial problems and source of referral. Our findings provide insight into the multifacetedness of parental motivation prior to starting treatment and inform health professionals of specific contextual factors of interest in parents' readiness to change their behaviour and participate in treatment.
\end{abstract}

Keywords Parental pre-treatment motivation $\cdot$ Child and adolescent psychiatry $\cdot$ LASSO regression $\cdot$ Behavioural problems $\cdot$ Emotional problems

\section{Introduction}

Parental motivation for treatment in child and adolescent mental health outpatient clinics is increasingly recognised as an important prerequisite for treatment success and for

Pieter J. Hoekstra and Andrea Dietrich shared last authorship

Electronic supplementary material The online version of this article (https://doi.org/10.1007/s00787-019-01391-9) contains supplementary material, which is available to authorized users.

Halewijn M. Drent

h.drent@accare.nl

1 Department of Child and Adolescent Psychiatry, University Medical Center Groningen, University of Groningen, Hanzeplein 1 A10, 9700 GZ Groningen, The Netherlands

2 Department of Cognitive Neuroscience, Donders Institute for Brain Cognition and Behaviour, Radboud University Medical Center, Nijmegen, The Netherlands preventing treatment dropout [1-7]. Treatment motivation may be conceptualized as readiness to change one's behaviour and willingness to participate in treatment $[1,6,8,9]$, but also facilitating the child in getting to appointments or using prescriptions [10]. A number of studies have focused on engagement of parents during treatment (e.g., barriers to treatment, attendance rates, or dropout) [11-13]. Especially the transtheoretical model of behavior change [14, 15] shows the different ways in which treatment engagement may change throughout the help-seeking and help-receiving process and, in addition, the importance of treatment engagement in child and adolescent mental healthcare [16]. However, little attention has been given to pre-treatment motivation, defined as parental readiness to start treatment (e.g., readiness to change one's behaviour, desire for change, perceived ability to change), and to the individual (e.g., patient's gender, age or problem severity) and contextual factors (e.g., source of referral, parental characteristics, social network strength) that are related to it. Addressing 
these individual and contextual factors may foster the identification of particularly vulnerable families characterised by lower motivation for treatment in child and adolescent mental health care and facilitate tailored interventions to increase treatment motivation in those families who are newly referred to a child and adolescent outpatient mental health clinic for emotional and/or behavioural problems of their child.

Previous studies identified a number of parent characteristics associated with parents' pre-treatment motivation. In a study among 386 families who took part in an intervention for children at risk for behavioural problems, a higher level of parental depression was related to higher levels of motivation (i.e., desire to change) of these parents to change their parenting techniques [17]. Also, higher parental stress was related to a higher level of parental pre-treatment motivation (i.e., treatment readiness, readiness to change) [18, 19]. Furthermore, poorer parenting skills (i.e., higher levels of inconsistent disciplining and poor supervision) were associated with higher parental motivation (i.e., treatment readiness) for receiving parenting treatment for parents of children with behavioural problems [20].

These studies also investigated child factors in relation to parents' pre-treatment motivation. Higher symptom severity was found to be related to higher parental pre-treatment motivation, as reported in a study among 197 adolescents when entering home-based treatment services for a variety of mental health problems [18] and in two studies of children who were referred to a mental health clinic for behavioural problems [19, 20].

The sparse literature about factors related to parents' treatment motivation prior to starting treatment in child and adolescent mental health care has important shortcomings: studies often consisted of small sample sizes, considered only a limited number of factors and focused predominantly on children with externalising problem behaviour rather than on the broad scope of problems of children and adolescents referred to mental health services [3, 17, 19-21]. Also, weaknesses in the various measures of motivation for treatment may be noted, e.g., rating scales with low internal consistency, a low number of items or open-ended questions $[3,17,21]$.

In the current study, we addressed the relation of a wide range of individual (i.e., child and parent) and contextual factors (i.e., family and environmental factors) with parents' pre-treatment motivation in a large sample of children and adolescents newly referred to one of the participating child and adolescent outpatient mental health clinics in this study. To assess parental pre-treatment motivation, we used the composite score of a validated parent rating scale that conceptualizes three aspects of motivation: desire for change, readiness to change and perceived ability to change [5]. Potential predictors were divided into five domains: (1) source of referral and prior use of health services, (2) child characteristics, (3) characteristics of the primary parent, (4) parenting characteristics of the primary parent and (5) family characteristics. In line with abovementioned studies, we expected higher parental stress and worse parental mental health (i.e., depression and anxiety), poorer parenting techniques and more severe child problems to be related to higher parental pre-treatment motivation. Furthermore, we investigated whether other individual and contextual factors were related to parental pre-treatment motivation.

\section{Method}

\section{Participants}

Our study included 521 families who participated in the baseline assessment of a three-wave survey study on the influence of child, family and environmental factors on response to treatment in outpatient child and adolescent mental health services. Families of children up to age 18 years $(97.1 \%$ with the primary parent of Dutch origin) were recruited from two large child and adolescent psychiatry centres with several locations in the Northern and Eastern part of the Netherlands, including both rural and urban areas. Children had to be newly referred (i.e., first referral to the respective mental healthcare clinic) to be eligible to participate in the study. Children referred for eating disorders or forensic problems were not invited to the study, because treatment trajectories in these settings are not always entirely voluntary, making pre-treatment motivation different from voluntary participants. The primary parent (i.e., the parent most involved in raising the child) and the child ( $\geq 8$ years old) were asked to fill out a set of questionnaires at each of the three waves related to a variety of contextual factors. Completion of questionnaires by the secondary parent (i.e., the partner of the primary parent living in the same household; in $82.9 \%$ of the families this was a biological parent of the child) was optional and only at baseline. In the present study, we used the baseline data of the primary parent, except for socio-economic status which also included data provided by the secondary parent. Participation was voluntary and families were rewarded with a voucher of 20 euros for participation in each study wave. It should be noted that mental healthcare in the Netherlands is accessible to all families. There were no incentives for a family to follow treatments, which were all voluntary.

\section{Procedures}

Figure 1 describes the sampling procedure of the baseline assessment. An invitation to participate in the study was sent along with the invitation for the first clinic visit by mail to 


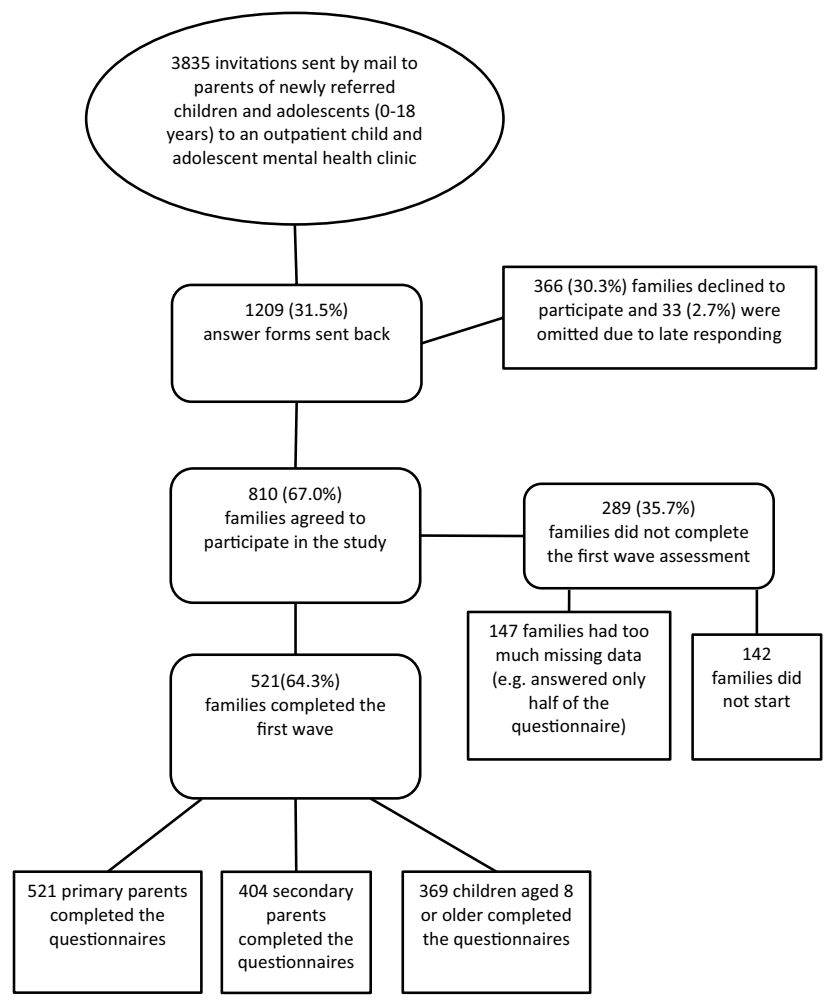

Fig. 1 Participation flow of the baseline assessment of a three-wave study on the influence of child, family and environmental factors on response to treatment in outpatient child and adolescent mental healthcare

all newly referred families between 1st May 2015 and 30th June 2016. Families who agreed to participate received personal login codes for the primary parent, secondary parent and child ( $\geq 8$ years old) to a set of online questionnaires via mail before a possible start of treatment. Twenty-one families received a paper version of the questionnaires. On average, families completed the questionnaire within 18 days after the personal login codes were sent (with a range of 1 day-3 months). E-mail reminders to respond to our invitation were sent 2 and 3 weeks after the personal login codes were sent. Families who still did not respond after the second e-mail reminder were approached by telephone (at least three calls made on different days and times). Families who agreed to participate or started the questionnaire after the 30th of June 2016 were omitted from the sample. Based on information from the electronic data entry system, completion of the baseline questionnaires took the primary parents about $75 \mathrm{~min}(n=512)$, the secondary parent about $30 \mathrm{~min}$ $(n=404)$ and the child about $20 \mathrm{~min}(n=369)$. Participants were able to pause answering the online questionnaire, but they were asked to complete the full set of questionnaires within 1 week after they started. Lastly, families were asked to complete the questionnaires before a possible start of treatment at the outpatient centres.

\section{Measures}

\section{Outcome measure}

Parental pre-treatment motivation was measured by the Parent Motivation Inventory (PMI), showing good validity and reliability [5, 22]. Parents rated 25 items on a five-point scale $(1=$ strongly disagree, $5=$ strongly agree; observed Cronbach's $\alpha=0.93$ ) addressing the need to change (e.g., 'My child's behaviour has to improve soon'), readiness to change ('I am willing to work on changing my own behaviour as it relates to managing my child') and perceived ability to change ('I believe that my child's behaviour cannot change without my involvement in treatment'); note that one item was removed from the scale (i.e., 'I am motivated to change the way I reward and punish my child if it will lead to improvement'), because there were too many missing responses on this item due to a technical difficulty in the online questionnaire. The PMI is a valid measure tested in a clinical sample of parents of American children with externalising problem behaviour $(\alpha=0.86)$ [5]. Furthermore, construct validity of the PMI is tested by the correlation with the credibility and expectancy questionnaire [22]. A higher score on the PMI indicates a higher level of parental pre-treatment motivation.

\section{Predictors}

Predictors of parental pre-treatment motivation were distinguished in five domains as described below. We used the total scale scores of questionnaires where appropriate. A detailed description of the scales can be found in Online Resource 1. Only data of the primary parents were used, unless otherwise noted.

Source of referral and prior use of healthcare services was measured by asking the primary parent who had initiated the help-seeking process: (1) parent(s) or child, (2) school, or (3) a health professional (e.g., general practitioner or hospital consultant). To assess the child's prior use of mental health services, the primary parent was asked whether the child had received some form of prior help for emotional and/or behavioural problems. Also, we assessed current medication use of the child via an open-ended question. Answers were dichotomised into 'No medication use' and 'Medication use'; both non-psychotropic as well as psychotropic medication were included.

Seven child characteristics were assessed: (1) gender, (2) age, (3) presence of learning difficulties or a mental disability, (4) general functioning at school, as indicated by a total score based on five self-constructed questions that capture satisfaction with school and school attendance; a higher score implies better functioning at school, (5) severity of internalising problems and (6) of externalising problems, 
both measured by the respective subscales of the parents' version of the Strength and Difficulties Questionnaire (SDQ; $[23,24])$; and (7) callous and unemotional traits; as assessed by the parent version of the Inventory of Callous-Unemotional Traits (ICU) [25, 26], with higher scores on SDQ and ICU indicating more severe problems or traits.

Characteristics of the primary parent included four predictors: (1) gender, (2) age, (3) current mental health (i.e., depressive and anxiety symptoms) as measured by the selfreported Mental Health Index-5 (MHI) [27]; a higher score indicates better mental health and (4) prior or current help for mental health problems, by asking whether the primary parent received some kind of help for mental health problems, currently or in the past.

Parenting characteristics were assessed with eight measures. Five subscales of the Alabama Parenting Questionnaire (APQ) [28, 29] were used: (1) involved parenting, (2) positive parenting, (3) poor monitoring, (4) inconsistent disciplining and (5) corporal punishment. Furthermore, (6) the General Self-Efficacy scale (GSE) [30] was used to measure the primary parent's perceived general self-efficacy; a higher score reflects a higher level of perceived general self-efficacy, (7) perceived parenting competence was measured by the Parenting Sense of Competence scale (PSOC) [31]; a higher score indicates a higher level of perceived parenting competence and (8) parental stress of the primary parent was assessed with the Parental Stress Scale (PSS) [32]; a higher score points to more parental stress.

Family characteristics were investigated by eight family factors: (1) single parent household, (2) presence of other children in the household, (3) socio-economic status, as assessed through a standardised composite score of the net income level of the household and the educational and occupational level of both parents (data from the secondary parent was also used) [33], based on the International Standard Classification of Occupations [34], (4) presence of financial problems, by asking the primary parents whether the monthly net income was sufficient (yes/no/ refused to answer), (5) urbanicity, based on the postal code through which participants were classified as living in a small-sized city ( $<40,000$ inhabitants), medium-sized city $(40,000-100,000$ inhabitants) or large-sized city $(>100,000$ inhabitants), (6) social network strength; defined by a mean composite score of seven standardised variables (e.g., number of contacts with friends and family, frequency of the contact); a higher score points to a stronger social network strength, (7) families with high-risk behaviours; assessed through a composite score of 22 'risk' variables (e.g., use of drugs, parental psychopathology, parental criminal record), a higher score indicating more high-risk behaviour of the family and (8) family functioning, using the Dutch Parental Questionnaire Family Functioning (VGFO) [35]; a higher score on the VGFO indicates a better family functioning regarding basic care for the child, nurture, social contacts of the primary parent, own youth experiences and partner relation of the primary parent.

\section{Statistical analysis}

To investigate which variables were associated with parental pre-treatment motivation, we applied the least absolute shrinkage and selection operator (LASSO) [36] using the glmnet-package in R $[37,38]$. This method selects the best fitting predictors by automatically assigning a penalized term to the standardised predictors. The selection of the penalty term is done by cross-validation, where the average mean cross-validated error is calculated through 10,000 iterations to get the best fitting penalty term. Since LASSO automatically assigns the penalty term to the predictors with a low error, this method is suitable for selecting the best set of predictors out of a large model. Assumptions of linearity, independence and constant variance of residuals were not violated. Prior to analysis, 15 missing cases on urbanicity (2.88\%) were imputed with 5 imputations by applying predictive mean matching via the mice-package in $\mathrm{R}[38,39]$. Furthermore, we tested for outliers with the Mahalanobis distance [40]; no outliers were found.

Six models were run to identify the best fitting predictors for parental treatment motivation. We started with five separate models on our five predictor domains: (1) source of referral and prior use of healthcare services, (2) child characteristics, (3) characteristics of the primary parent, (4) parenting characteristics of the primary parent and (5) family characteristics. In the final overall model, all statistically relevant predictors were included. Statistical relevance of a predictor was defined as the presence of a non-zero beta coefficient and non-zero explained deviance (comparable to explained variance, but based on the $-2 \log$-likelihood instead of the residuals of the model). The explained deviance of the predictors is calculated through the leave-one-out method; the predictor of interest is left out of the total model causing a change in the explained deviance of the total model. The difference between the explained deviance of the total model and the model without the predictor of interest is the contribution of this predictor to the model [41].

\section{Results}

\section{Sample characteristics}

Table 1 shows the descriptive statistics of all study variables. Ethnicity of the primary parent was not included as a predictor due to the homogeneous sample; $97.1 \%$ of primary parents were Dutch. Mothers generally were the primary parents (85\%). The mean age of the children was 10.2 years 
Table 1 Descriptive statistics of all study variables obtained from the primary parent $(n=521)$

\begin{tabular}{|c|c|c|c|}
\hline & Mean, $n$ & $\begin{array}{l}\text { Standard devia- } \\
\text { tion, } \%\end{array}$ & Range \\
\hline \multicolumn{4}{|l|}{ Outcome measure } \\
\hline Treatment motivation of the primary parent (PMI) & 3.80 & 0.53 & $1-5$ \\
\hline \multicolumn{4}{|l|}{ Source of referral and prior use of healthcare services } \\
\hline \multicolumn{4}{|l|}{ Source of referral } \\
\hline Parent(s) or child & 326 & 62.6 & \\
\hline School & 89 & 17.1 & \\
\hline Health professional & 106 & 20.3 & \\
\hline Prior use of mental health services for the child & 235 & 45.1 & \\
\hline Current medication use by the child & 110 & 21.1 & \\
\hline \multicolumn{4}{|l|}{ Child characteristics } \\
\hline Female gender & 207 & 39.7 & \\
\hline Age (years) & 10.2 & 3.76 & $1-18$ \\
\hline Presence of learning difficulties or mental disability & 200 & 38.4 & \\
\hline General functioning at school ${ }^{\mathrm{a}}$ & 3.93 & 0.75 & $1-5$ \\
\hline Severity of internalising problems (SDQ) & 1.80 & 0.39 & $1-3$ \\
\hline Severity of externalising problems (SDQ) & 1.91 & 0.41 & $1-3$ \\
\hline Callous and unemotional traits (ICU) & 2.27 & 0.43 & $1-4$ \\
\hline \multicolumn{4}{|l|}{ Characteristics of the primary parent } \\
\hline Female gender & 443 & 85.0 & \\
\hline Age (years) & 41.2 & 6.47 & $25-65$ \\
\hline Current mental health (MHI-5) ${ }^{\mathrm{a}}$ & 2.34 & 0.83 & $1-6$ \\
\hline $\begin{array}{l}\text { Prior or current help for mental health problems of the } \\
\text { primary parent }\end{array}$ & 255 & 48.9 & \\
\hline \multicolumn{4}{|l|}{ Parenting characteristics of the primary parent } \\
\hline Involved parenting (APQ) $)^{\mathrm{a}}$ & 3.87 & 0.45 & $1-5$ \\
\hline Positive parenting $(\mathrm{APQ})^{\mathrm{a}}$ & 3.96 & 0.50 & $1-5$ \\
\hline Poor monitoring (APQ) & 2.38 & 0.43 & $1-5$ \\
\hline Inconsistent disciplining (APQ) & 2.47 & 0.54 & $1-5$ \\
\hline Corporal punishment (APQ) & 1.57 & 0.45 & $1-5$ \\
\hline Perceived general self-efficacy $(G S E)^{\mathrm{a}}$ & 3.08 & 0.58 & $1-4$ \\
\hline Perceived parenting competence (PSOC) ${ }^{\mathrm{a}}$ & 4.29 & 0.66 & $1-5$ \\
\hline Parental stress (PSS) & 2.03 & 0.49 & $1-6$ \\
\hline \multicolumn{4}{|l|}{ Family characteristics } \\
\hline Single parent household & 113 & 21.7 & \\
\hline Presence of other children in the household & 439 & 84.3 & \\
\hline \multicolumn{4}{|l|}{ Socio-economic status of the family ${ }^{\mathrm{b}}$} \\
\hline Low & 67 & 12.9 & \\
\hline Intermediate & 391 & 75.0 & \\
\hline High & 63 & 12.1 & \\
\hline \multicolumn{4}{|l|}{ Presence of financial problems } \\
\hline Yes & 367 & 70.4 & \\
\hline No & 109 & 20.9 & \\
\hline Refused to answer & 45 & 8.6 & \\
\hline \multicolumn{4}{|l|}{ Urbanicity } \\
\hline Small-sized city $(<40,000$ inhabitants $)$ & 239 & 45.9 & \\
\hline Middle-sized city (40,000-100,000 inhabitants) & 160 & 30.7 & \\
\hline Large-sized city ( $>100,000$ inhabitants) & 122 & 23.4 & \\
\hline \multicolumn{4}{|l|}{ High-risk behaviour families } \\
\hline Low-risk families & 75 & 14.4 & \\
\hline Normal risk families & 365 & 70.1 & \\
\hline
\end{tabular}


Table 1 (continued)

\begin{tabular}{cllc}
\hline & Mean, $n$ & $\begin{array}{l}\text { Standard devia- } \\
\text { tion, } \%\end{array}$ & Range \\
\hline $\begin{array}{c}\text { High-risk families } \\
\text { Family functioning }(\mathrm{VGFO})^{\mathrm{a}}\end{array}$ & 81 & 15.5 & $1-4$ \\
\hline
\end{tabular}

See Online Resource 1 for psychometric properties of the scales

$P M I$ parental motivation inventory [5], $S D Q$ Strength and Difficulties Questionnaire [18], ICU inventory of callous-unemotional traits [20], MHI-5 Mental Health Index-5 [22], APQ Alabama Parenting Questionnaire [23], GSE general self-efficacy [25], PSOC Parental Sense of Competence Scale [26], PSS Parental Stress Scale [27], VGFO Parental Questionnaire Family Functioning [30]

${ }^{a} \mathrm{~A}$ higher score indicates better functioning, mental health or parenting

${ }^{\mathrm{b}}$ Includes data from the secondary parent where available

(range 1-18 years) and about $60 \%$ were boys. About onefifth of the children lived in a single parent household. Approximately, $13 \%$ of the families were from a low socioeconomic background and almost half of the families resided in a small-sized city ( $<40,000$ inhabitants). Furthermore, about half of the children had received some kind of professional help for their problems in the past and one-fifth of the children currently used medication.

\section{LASSO regression}

Table 2 shows the explained deviances and beta coefficients for the various predictors of the five domain-specific models and the overall model with all relevant predictors based on LASSO regression analyses. As explained, predictors are statistically relevant if they were not shrunk to zero (i.e., beta coefficient and explained deviance were higher than zero).

\section{Source of referral and prior use of healthcare services}

Model 1 showed a total explained deviance of $4.0 \%$. All four variables were relevant predictors in the model; source of referral contributed the most. Both school $(1.72 \%$; $\beta=-0.14)$ and a health professional $(1.42 \% ; \beta=-0.12)$ as source of referral were related to a lower level of parental pre-treatment motivation, in comparison to self-referral by parent(s)/child. Moreover, current use of medication by the child was related to a lower level of parental motivation $(0.39 \% ; \beta=-0.06)$. Lastly, prior use of mental health services for the child was related to a higher level of parental motivation $(0.86 \% ; \beta=0.09)$.

\section{Child characteristics}

The total explained deviance of Model 2 was $12.1 \%$. Five of the seven variables were relevant predictors of parental pre-treatment motivation. Severity of internalising problems $(4.77 \% ; \beta=0.21)$ was the strongest contributor to the model, followed by externalising problems $(1.55 \% ; \beta=0.14)$ and callous and unemotional traits $(0.77 \% ; \beta=0.08)$, all related to a higher level of parental pre-treatment motivation. In contrast, child's older age $(1.49 \% ; \beta=-0.12)$ and the presence of learning difficulties or a mental disability $(0.10 \%$; $\beta=-0.02$ ) were related to a lower level of parental motivation. However, gender of the child and general functioning at school were non-relevant factors with both beta values and explained deviances shrunk to zero.

\section{Characteristics of the primary parent}

Model 3 showed a total explained deviance of $2.59 \%$. Three of the four characteristics of the primary parent remained relevant in the model, namely (1) parent's age, (2) current mental health and (3) prior or current help for mental health problems. The strongest predictor in the model was a better current mental health of the primary parent $(0.66 \% ; \beta=0.08)$, followed by receiving prior or current help for mental health problems of the primary parent $(0.56 \% ; \beta=0.07)$, all related to a higher level of parental pre-treatment motivation. In contrast, older primary parents had a lower level of pre-treatment motivation $(0.52 \%$; $\beta=-0.06)$, while the primary parent's gender was not a relevant predictor.

\section{Parenting characteristics of the primary parent}

Model 4 showed a total explained deviance of 5.93\%. Four of the eight predictors were relevant predictors for parental pre-treatment motivation: the strongest predictor was a higher perceived parenting competence $(\beta=-0.22)$ related to a lower level of parental motivation and explaining $2.87 \%$ of its deviance. Furthermore, a higher level of corporal punishment $(0.35 \% ; \beta=-0.04)$ and poor monitoring $(0.12 \%$; $\beta=-0.01)$ were related to a lower level of parental motivation. In contrast, a higher perceived general self-efficacy emerged as the second important predictor, which was related to a higher level of parental pre-treatment motivation $(1.78 \% ; \beta=0.11)$. Four predictors were not relevant predictors, namely (1) involved parenting, (2) positive parenting, (3) inconsistent disciplining and (4) parental stress. These 


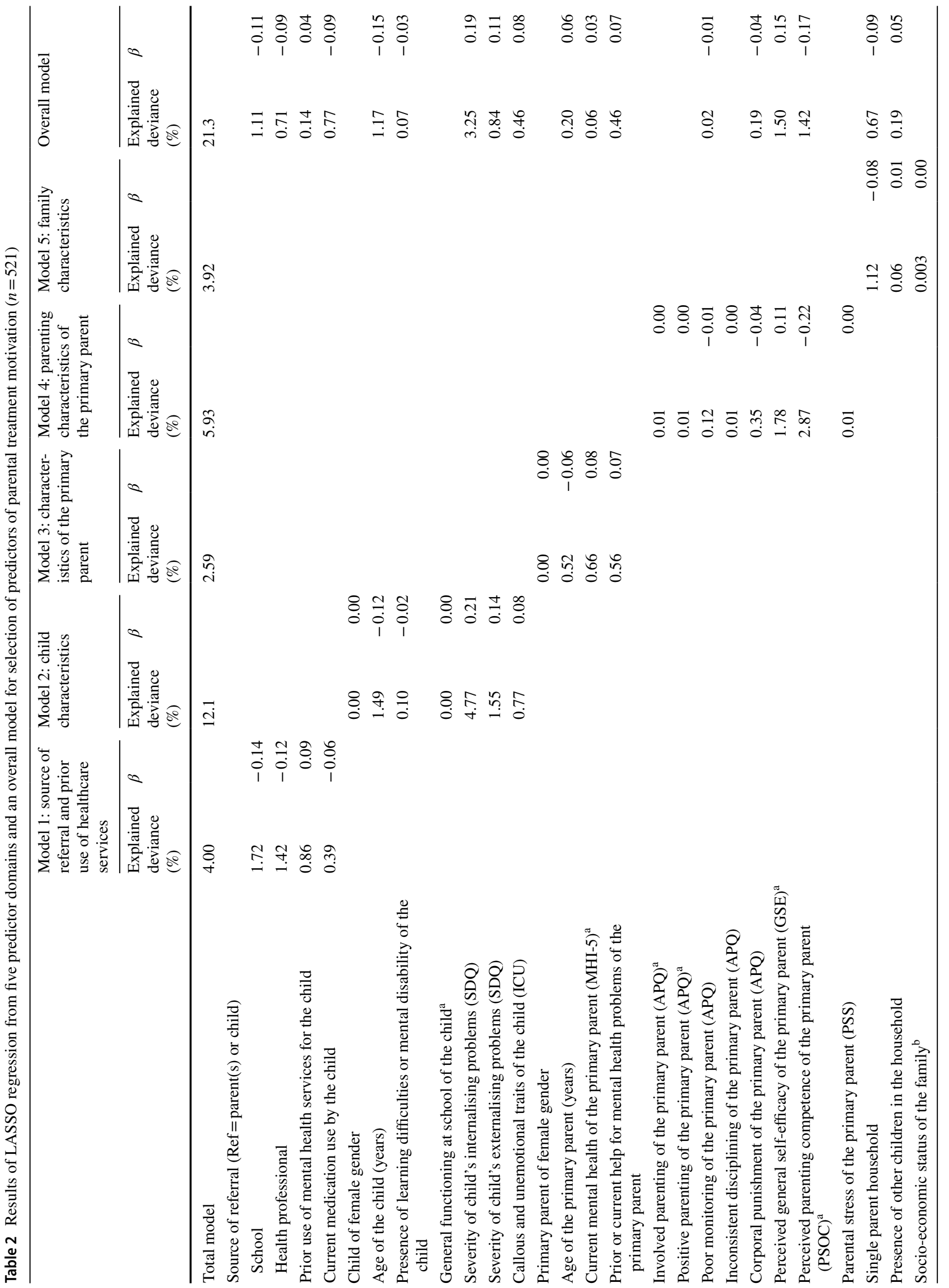




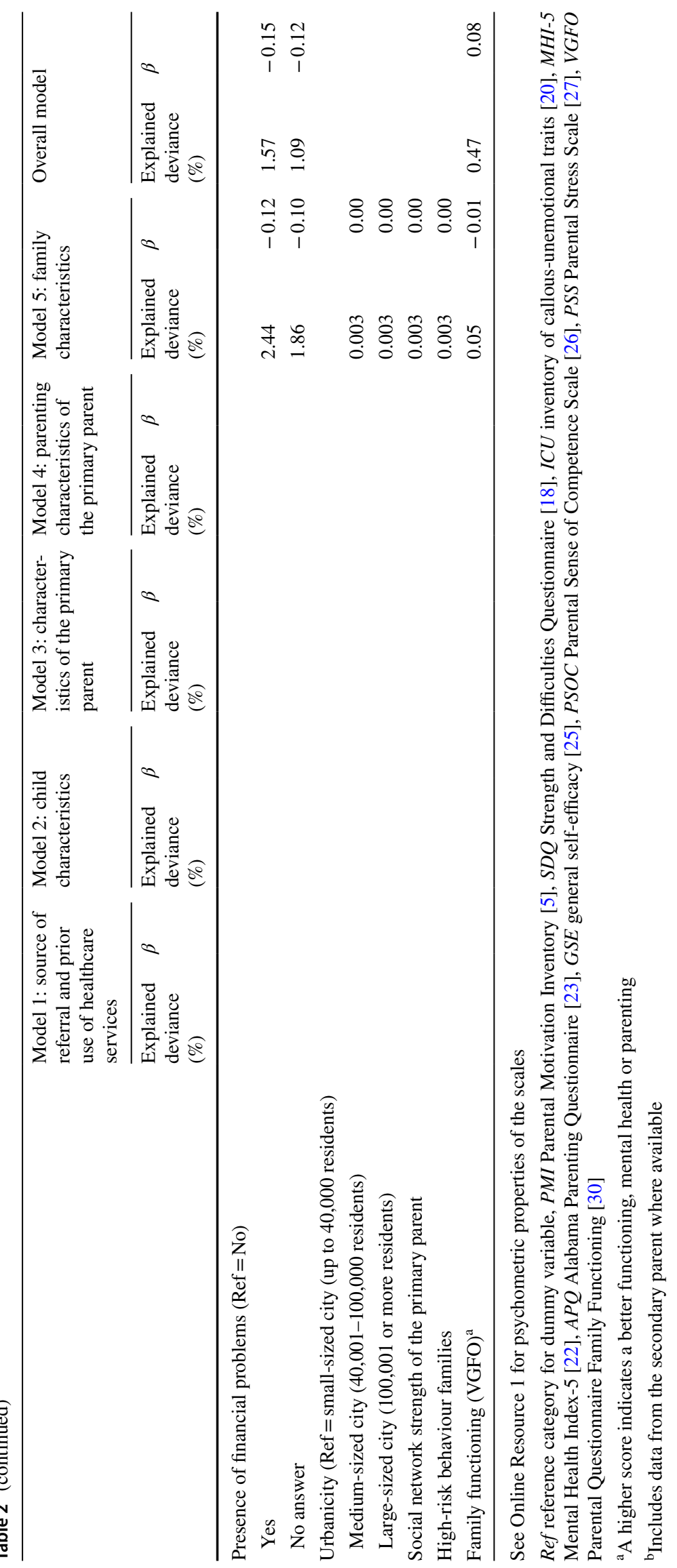


predictors explained a small percentage of deviance in pretreatment motivation, most likely due to a shared contribution of the different predictors to the model.

\section{Family characteristics}

The predictors of the family characteristics domain explained a total of $3.92 \%$ of the deviance, with in total five out of ten predictors being relevant. The strongest relation was found for the presence of financial problems. Both the presence of financial problems $(2.44 \% ; \beta=-0.12)$ and those who refused to answer whether there were financial problems $(1.86 \% ; \beta=-0.10)$ were related to a lower parental pre-treatment motivation, in comparison to the absence of financial problems. Moreover, having a single parent household $(1.12 \% ; \beta=-0.08)$ and better family functioning $(0.05 \% ; \beta=-0.01)$ were related to a lower level of parental motivation. In contrast, the presence of other children in the household was related to a higher parental motivation $(0.06 \% ; \beta=0.01)$. Non-relevant factors were: (1) socio-economic status, (2) urbanicity, (3) social network strength and (4) high-risk behaviour families; although a small portion of explained deviance was found, this is most likely due to the shared contribution of different predictors to the model.

\section{Overall model}

All statistically relevant predictors of the five different predictor domains were entered into one overall model. In total, 21 predictors were entered and all remained relevant, explaining a total of $21.3 \%$ of primary parents' pre-treatment motivation. To compare the relative strength of the different domains, we summed the explained deviances of the individual predictors per domain. We found the highest explained deviance for child characteristics (5.79\%), followed by family characteristics $(3.99 \%)$, parenting characteristics of the primary parent (3.13\%), source of referral and prior use of healthcare services $(2.79 \%)$ and, lastly, characteristics of the primary parent $(0.79 \%)$.

The strongest predictor related to a higher parental pretreatment motivation was the severity of the child's internalising problems $(3.25 \% ; \beta=0.19)$, and to a lesser extent the externalising behavioural domain (i.e., externalising problems and higher callous-unemotional traits together explaining $1.30 \%$ ), followed by perceived general self-efficacy of the primary parent $(1.50 \% ; \beta=0.15)$. In contrast, the strongest predictor related to a lower parental pre-treatment motivation was families' financial problems and refusal to answer whether there were financial problems (together explaining $2.66 \%$ ). This was followed by referral to the outpatient clinic prompted by a school or health professional (together explaining $1.82 \%$ ) rather than by the parent or child, a higher perceived parenting competence $(1.42 \% ; \beta=-0.17)$ and an older age of the child $(1.17 \% ; \beta=-0.15)$. For other predictors we refer to Table 2 .

In the overall model, two factors were noted to change in direction of effects, i.e., higher age of the primary parent (Model 3: $0.52 \% ; \beta=-0.06$ ) and better family functioning (Model 5: $0.05 \% ; \beta=-0.01$ ) were no longer related to a lower level of parental motivation, but to a higher level of motivation $(0.20 \% ; \beta=0.06$ and $0.47 \% ; \beta=0.08$, respectively).

\section{Discussion}

The aim of this study was to identify factors related to parents' pre-treatment motivation of children and adolescents who had been newly referred to an outpatient child and adolescent mental health clinic. For any treatment to be successful, there is a large dependency on the parents' willingness to participate in treatment and their readiness to change their own behaviour; regarding behavioural interventions, but also daily organization and possible lifestyle changes $[1,6,8,9]$. To our knowledge, this is the first study that investigated a large set of individual and contextual factors in relation to parental motivation before the start of treatment across five domains using LASSO regression. We assessed the role of (1) source of referral and prior use of healthcare services, (2) child characteristics, (3) characteristics of the primary parent, (4) parenting characteristics of the primary parent and (5) family characteristics. Notably, our overall model was able to explain more than one-fifth of the primary parent's pre-treatment motivation based on these individual and contextual variables. While we were able to explain a sizeable portion of parents' motivation, it should be noted that a total of 21 individual variables emerged as relevant factors from the model, each of them showing a small contribution. This points to a large heterogeneity in factors that may affect parents' motivation and highlights its multifactorial nature. Child characteristics contributed the most, while, surprisingly, characteristics of the primary parent explained little of parental pre-treatment motivation. The most important factors were the severity and type of the child's problems, financial problems of the family, source of referral to the clinic, the perceived self-efficacy of the primary parent, parenting competence of the primary parent and age of the child. In the following, we will discuss only the most relevant factors (explained deviance $\geq 1 \%$ ).

We started by investigating factors related to the source of referral and child's prior use of mental healthcare. Our study indicates that parents' pre-treatment motivation is higher when family members themselves decided to reach out for help, rather than when school or health professionals were the primary source of referral. Especially, when the child was referred by the school, the parental motivation to start 
treatment was lower. An explanation might be that problems arising at school might not be present or apparent at home and, thus, a parent disagrees with the school's view that their child is in need of behavioural treatment of medication for emotional or behavioural problems.

Regarding child characteristics, the severity of a child's problems was most strongly linked to parental pre-treatment motivation. This is consistent with previous findings [18-20] where higher symptom severity was related to a higher parental motivation to start treatment. Notably, we found the strongest relation for internalising problems, while the main focus of previous studies was on externalising problem behaviour $[19,20]$. Our finding is somewhat surprising since our measure of parental motivation focusses on actively changing a parent's own behaviour, which appears to be more relevant to the child's externalising than to internalising problems. We assume that a child's internalising problems may perhaps be more threatening or alarming to parents than a child's externalising problems and callous-unemotional traits. Parents may also feel a greater sense of helplessness and lack of understanding of their child's anxious and depressive symptoms. These aspects may increase the willingness to receive treatment and change parental behaviour. We are not aware of existing studies that investigated this link, which makes it an interesting topic for future research. In contrast, parents of older children or adolescents had a lower level of pre-treatment motivation, likely reflecting the normal developmental course with lower involvement from parents of older children or adolescents and the greater availability of therapeutic interventions tailored primarily for child or adolescent participation.

Subsequently, we considered characteristics of the primary parent. In contrast to Fosco et al. [17], we found that parents with a better current mental health showed higher pre-treatment motivation. This suggests that parents with a lesser degree of depressive or anxiety symptoms are more capable to support their child in need of mental health care. A possible explanation for this difference in findings is that we did not specifically look at a certain type of problem, while the abovementioned authors only looked at children with antisocial behaviour.

With respect to parenting characteristics of the primary parent, surprisingly, we found that higher perceived parenting sense of competence was related to a lower level of parents' pre-treatment motivation, while a higher perceived selfefficacy (e.g., capability of coping with a variety of common demands in life) of the primary parent was associated with a higher treatment motivation. This may appear somewhat contradictory, since parenting sense of competence can be seen as a part of general self-efficacy. An explanation might be that parents who believe that they are competent parents are less inclined to change their parental behaviours due to treatment, while parents with an overall high self-efficacy do want change to happen and believe they can contribute to their child's treatment. We expected parenting style to be of influence on parental motivation as well. For instance, Andrade et al. [20] found higher levels of inconsistent disciplining and poor monitoring to be related to a higher level of parental motivation. We did not find a strong relation and it seems more likely that poorer parenting skills are related to a lower level of motivation. Furthermore, our study did not confirm a role for parenting stress previously shown to be related to a higher level of parental pre-treatment motivation $[18,19]$, perhaps explained by the large number of variables in our model that may have been more important (e.g., perceived self-efficacy, perceived parenting competence).

In our final domain, we investigated family characteristics. We found a notable association between presence of financial problems (and refusal to answer that question) and lower level of parents' pre-treatment motivation but not, as expected, with socio-economic status (based on family income, educational level and occupation of the primary parent and the partner). Furthermore, single parent household contributed, to a lesser extent, to lower parental motivation, independent from financial problems. It thus appears that the broader domain indicating socio-economic position was more important in this model than the classic composite score indicating socio-economic status. Moreover, better family functioning and more children in the household were related to higher parental pre-treatment motivation, however, effects were minor. In contrast to our expectations, social support and high-risk family status (e.g., parental substance use, contact with judicial system) were unrelated to parental pre-treatment motivation.

Strengths of our study were the use of LASSO regression to investigate a wide variety of potential factors of parental pre-treatment motivation, using a well-validated measure, in a large sample of parents and their children newly referred to an outpatient child and adolescent mental health care setting. Although LASSO regression has the limitation that it randomly selects one predictor if factors are correlated [42], we minimized this by running the model 10,000 times to select the best error term in a similar way to the stability selection technique described by Meinshausen and Bühlmann [43]. A limitation might be selection bias; due to the lack of data on families who did not respond to our anonymized study invitation, we were unable to investigate this. Study participants will most likely show higher levels of motivation for treatment than the average referred family at a mental health clinic who did not participate in the study. Therefore, factors that are related to low motivation may be underestimated in our study. Findings should also be considered in light of our study population including a wide variety of mental health problems of varying intensity and may not readily apply to speciality clinics focusing on more severely affected patient groups. However, by including two large 
child and adolescent psychiatric outpatient centres with different locations, we ensured a large catchment area covering about one-third of the Netherlands. Furthermore, our sample consisted largely of parents of Dutch origin, hence findings may not be generalisable to other ethnicities or minority groups. Finally, we acknowledge that youths' motivational factors, not addressed in our study, are also an important area of research.

We conclude that mainly child characteristics (severity and type of problems), socio-demographic factors (financial problems and referral through school or health professionals) and parenting characteristics (perceived self-efficacy and parenting competence) are important factors of parental pre-treatment motivation. Since parents' readiness to participate in treatment and motivation to bring about change are essential in facilitating successful treatment, health professionals should pay particular attention to these individual and contextual factors early during clinical counselling of newly referred patients in a child and adolescent outpatient setting. This will help in identifying particularly vulnerable families characterised by lower motivation for treatment, setting off more targeted strategies to increase treatment motivation in those families. Future research is needed to see how contextual factors are related to response to treatment and how treatment motivation is related to other barriers (e.g., stigma, therapy related problems) in the help-seeking process and during the course of treatment in child and adolescent mental health care.

Acknowledgements This work was supported by the ZonMw Grant 729101011. The funding agency was not involved in the design of the study, the collection of the data, analyses and interpretation, or the preparation, review or approval of the manuscript.

\section{Compliance with ethical standards}

Conflict of interest On behalf of all authors, the corresponding author states that there is no conflict of interest.

Open Access This article is distributed under the terms of the Creative Commons Attribution 4.0 International License (http://creativeco mmons.org/licenses/by/4.0/), which permits unrestricted use, distribution, and reproduction in any medium, provided you give appropriate credit to the original author(s) and the source, provide a link to the Creative Commons license, and indicate if changes were made.

\section{References}

1. King G, Currie M, Petersen P (2014) Review: Child and parent engagement in the mental health intervention process: a motivational framework. Child Adolesc Mental Health 19(1):2-8. https ://doi.org/10.1111/camh.12015

2. Lindsey MA, Brandt NE, Becker KD, Lee BR, Barth RP, Daleiden EL, Chorpita BF (2014) Identifying the common elements of treatment engagement interventions in children's mental health services. Clin Child Fam Psychol Rev 17(3):283-298

3. Miller GE, Prinz RJ (2003) Engagement of families in treatment for childhood conduct problems. Behav Ther 34(4):517-534. https ://doi.org/10.1016/S0005-7894(03)80033-3

4. Nock MK, Ferriter C (2005) Parent management of attendance and adherence in child and adolescent therapy: a conceptual and empirical review. Clin Child Fam Psychol Rev 8(2):149-166. https://doi.org/10.1007/s10567-005-4753-0

5. Nock MK, Photos V (2006) Parent motivation to participate in treatment: assessment and prediction of subsequent participation. J Child Fam Stud 15(3):345-358. https://doi.org/10.1007/s1082 6-006-9022-4

6. Staudt M (2007) Treatment engagement with caregivers of at-risk children: gaps in research and conceptualization. J Child Fam Stud 16(2):183-196. https://doi.org/10.1007/s10826-006-9077-2

7. Wergeland GJH, Fjermestad KW, Marin CE, Storm-Mowatt Haugland B, Silverman WK, Öst L-G et al (2015) Predictors of dropout from community clinic child CBT for anxiety disorders. J Anxiety Disord 31:1-10. https://doi.org/10.1016/j.janxdis.2015.01.004

8. Drieschner KH, Lammers SMM, Van Der Staak CPF (2004) Treatment motivation: an attempt for clarification of an ambiguous concept. Clinical Psychology Review 23(8):1115-1137. https ://doi.org/10.1016/j.cpr.2003.09.003

9. Morrissey-Kane E, Prinz RJ (1999) Engagement in child and adolescent treatment: the role of parental cognitions and attributions. Clin Child Fam Psychol Rev 2(3):183-198. https://doi. org/10.1023/A:1021807106455

10. Taub J, Tighe TA, Burchard J (2001) The effects of parent empowerment on adjustment for children receiving comprehensive mental health services. Children's Services 4(3):103-122. https://doi. org/10.1207/S15326918CS0403_1

11. Kazdin AE, Holland L, Crowley M, Breton S (1997) Barriers to Treatment Participation Scale: evaluation and validation in the context of child outpatient treatment. J Child Psychol Psychiatry 38(8):1051-1062. https://doi.org/10.1111/j.1469-7610.1997.tb016 21. $\mathrm{x}$

12. Haine-Schlagel R, Walsh NE (2015) A review of parent participation engagement in child and family mental health treatment. Clin Child Fam Psychol Rev 18(2):133-150. https://doi.org/10.1007/ s10567-015-0182-x

13. Gopalan G, Goldstein L, Klingenstein K, Sicher C, Blarke C, McKay MM (2010) Engaging families into child mental health treatment: updates and special considerations. J Can Acad Child Adolesc Psychiatry 19(3):182-196

14. O'Donohue W, Snipes C, James L (2017) Practical strategies and tools to promote treatment engagement. Pract Strateg Tools Promote Treat Engagem. https://doi.org/10.1007/978-3-319-49206-3

15. Prochaska JO, Velicer WF (1997) The transtheoretical model of health behavior change. Am J Health Promot 12(1):38-48. https ://doi.org/10.4278/0890-1171-12.1.38

16. Byrd MR, Kentor R, Perez M (2017) Promoting active treatment engagement in child and family therapy. In: O'Donohue W, James L, Snipes C (eds) Practical strategies and tools to promote treatment engagement. Springer, Cham, pp 291-307

17. Fosco GM, Van Ryzin M, Stormshak EA, Dishion TJ (2014) Putting theory to the test: examining family context, caregiver motivation, and conflict in the Family Check-Up model. Dev Psychopathol 26(2):305-318. https://doi.org/10.1017/S09545794130010 04

18. Breda CS, Riemer M (2012) Motivation for youth's treatment scale (MYTS): a new tool for measuring motivation among youths and their caregivers. Administration Policy Mental Health Mental Health Serv Res 39(1):118-132. https://doi.org/10.1007/s1048 8-012-0408-x 
19. Jones HA, Putt GE, Rabinovitch AE, Hubbard R, Snipes D (2017) Parenting stress, readiness to change, and child externalizing behaviors in families of clinically referred children. J Child Fam Stud 26(1):225-233. https://doi.org/10.1007/s10826-016-0547-x

20. Andrade BF, Browne DT, Naber AR (2015) Parenting skills and parent readiness for treatment are associated with child disruptive behavior and parent participation in treatment. Behav Ther 46(3):365-378. https://doi.org/10.1016/j.beth.2015.01.004

21. Becker KD, Mathis G, Mueller CW, Issari K, Atta SS, Okado I (2012) Barriers to treatment in an ethnically diverse sample of families enrolled in a community-based domestic violence intervention. J Aggress Maltreat Trauma 21(8):829-850. https://doi. org/10.1080/10926771.2012.708013

22. Nock MK, Ferriter C, Holmberg E (2007) Parent beliefs about treatment credibility and effectiveness: assessment and relation to subsequent treatment participation. J Child Fam Stud 16(1):2738. https://doi.org/10.1007/s10826-006-9064-7

23. Goodman R (1997) The Strengths and Difficulties Questionnaire: a research note. J Child Psychol Psychiatry 38(5):581-586. https ://doi.org/10.1111/j.1469-7610.1997.tb01545.x

24. Van Widenfelt BM, Goedhart AW, Treffers PDA, Goodman R (2003) Dutch version of the Strengths and Difficulties Questionnaire (SDQ). Eur Child Adolesc Psychiatry 12(6):281-289. https ://doi.org/10.1007/s00787-003-0341-3

25. Frick PJ (2004) Inventory of callous-unemotional traits. Unpublished rating scale. University of New Orleans, Orleans

26. Roose A, Bijttebier P, Decoene S, Claes L, Frick PJ (2010) Assessing the affective features of psychopathy in adolescence: a further validation of the inventory of callous and unemotional traits. Assessment 17(1):44-57. https://doi.org/10.1177/1073191109 344153

27. Berwick DM, Murphy JM, Goldman PA, Ware JE Jr, Barsky AJ, Weinstein MC (1991) Performance of a five-item mental health screening test. Med Care 29(2):169-176. https://doi. org/10.1097/00005650-199102000-00008

28. Frick PJ (1991) The Alabama Parenting Questionnaire. Unpublished rating scale. University of Alabama, Tuscaloosa

29. Van Lier PAC, Crijnen AAM (1999) Alabama Parenting Questionnaire, Nederlandse vertaling [Alabama Parenting Questionnaire, Dutch translation]. Erasmus MC: Unpublished manuscript

30. Schwarzer R, Jerusalem M (1995) Generalized Self-Efficacy scale. In: Weinman J, Wright S, Johnston M (eds) Measures in health psychology: a user's portfolio. Causal and control beliefs. NFERNELSON, Windsor, pp 35-37

31. Johnston C, Mash EJ (1989) A measure of parenting satisfaction and efficacy. J Clin Child Psychol. https://doi.org/10.1207/s1537 4424jccp1802_8

32. Berry JO, Jones WH (1995) The parental stress scale: initial psychometric evidence. J Soc Pers Relationsh 12(3):463-472 (doi: 0803973233)

33. Ganzeboom HBG, De Graaf PM, Treiman DJ (1992) A standard international socio-economic index of occupational status. Soc Sci Res 21(1):1-56. https://doi.org/10.1016/0049-089X(92)90017-B

34. International Labor Office (2012) International standard classification of occupations: ISCO-08. International Labor Office, Geneva

35. Veerman JW, Janssen J, Kroes G, Meyer R, de Nguyen L, Vermulst A (2006) Vragenlijst Gezinsfunctioneren volgens Ouders (VGFO). Nijmegen: Praktikon BV

36. Tibshirani R (1996) Regression shrinkage and selection via the Lasso. J R Stat Soc Ser B (Methodol) 1:267-288

37. Friedman J, Hastie T, Tibshirani R (2010) Regularization paths for generalized linear models via coordinate descent. J Stat Softw 33(1):1-22. https://doi.org/10.1016/j.expneurol.2008.01.011

38. R Core Team (2016) R: a language and environment for statistical computing. $\mathrm{R}$ foundation for statistical computing, Vienna, Austria. https://www.r-project.org/. Accessed 15 Mar 2018

39. van Buuren S, Groothuis-Oudshoorn K (2011) mice: multivariate imputation by chained equations in R. J Stat Softw 45(3):1-67. https://doi.org/10.18637/jss.v045.i03

40. Mahalanobis PC (1936) On the generalised distance in statistics. Proc Natl Inst Sci India 12:49-55

41. Bertocci MA, Bebko G, Versace A, Fournier JC, Iyengar S, Olino $T$ et al (2016) Predicting clinical outcome from reward circuitry function and white matter structure in behaviorally and emotionally dysregulated youth. Mol Psychiatry 21(9):1194-1201. https ://doi.org/10.1038/mp.2016.5

42. Zou H, Hastie $\mathrm{T}$ (2005) Regression and variable selection via the elastic net. J R Stat Soc Ser B (Stat Methodol) 67(2):301-320. https://doi.org/10.1111/j.1467-9868.2005.00503.x

43. Meinshausen N, Bühlmann P (2010) Stability selection. J R Stat Soc Ser B Stat (Methodol) 72(4):417-473 